\title{
Learning Communities im Web 2.0 am Beispiel von Methopedia - Inspirationen für die Lehre?
}

\author{
Margit Scholl, Christian Niemczik, Maika Büschenfeldt
}

\section{Zusammenfassung}

Was macht »Communities«, in denen Millionen von Menschen sich untereinander elektronisch via Internet austauschen, so attraktiv?

Definitionen »virtueller Gemeinschaften« betonen insbesondere den sozialen Zusammenschluss auf der Grundlage persönlicher Beziehungen im Cyberspace. Im folgenden Beitrag geht es um die Frage, wie das Phänomen der großen Akzeptanz solcher sozialer Netzwerke im Sinne von Bildung und lebensbegleitendem Lernen genutzt werden kann. Gerade der informelle Charakter von Communities bietet große Chancen für das Anstoßen von Lernprozessen in nicht formalen bzw. informellen Zusammenhängen und könnte damit von maßgeblicher Bedeutung für die Umsetzung des Ziels »lebenslanges Lernen« sein. Die Hochschulen sollten diesen Trend bei der Entwicklung ihrer Lernarrangements ernst nehmen und den Lehrenden Rahmenbedingungen ermöglichen, in denen die Integration von nicht formalem und informellem Lernen in die formalen Abläufe sichergestellt wird. Lernenden würde sich damit die Möglichkeit erschließen, systematisch auch nicht formale und informelle Lernformen zu praktizieren. Als Praxisbeispiel einer »Learning Community« von Lehrenden wird die wikibasierte Entwicklung von »Methopedia« vorgestellt, die sich selbst mit den Methoden des Lernens und Lehrens praxisorientiert auseinandersetzt. Mit dem »MethopediaLearning Design-Planer« können Lehrende Methopedia als Methodenpool für das Gestalten der eigenen Seminare nutzen.

\section{Abstract}

What makes «communities», in which millions of people interact with each other electronically via the Internet, so attractive?

Definitions of »virtual communities« emphasize the social concentration on the basis of personal relationships in cyberspace. We ask ourselves, how can the phenomenon of the great acceptance of such social networks be used within education and lifelong learning? Precisely the informal nature of communities offers many opportunities for engaging in learning processes of non-formal and informal contexts, and could thus be of crucial importance for the implementation of promoting lifelong learning. Universities should take this trend seriously and should give teachers a framework to ensure the integration of formal and informal learning into formal procedures. Learners would thus open up the possibility of a systematic way to practice not formal and informal learning. For the teachers we provide the support of the wiki-based development of »Methopedia« as a practical example of a »Learning Community«, which itself deals with the practical methods of learning and teaching. With the »Methopedia - Learning Design - Planner « teachers can use Methopedia as a pool of methods for designing their own seminars.

\section{Einleitung}

Ausgehend vom Begriff der virtuellen Community soll im Folgenden versucht werden auszuloten, inwieweit die aktuelle Praxis von Communities im Web 2.0 in der formalen Lehrvorbereitung, Lehrdurchführung und Lehrnachbereitung auch institutionell genutzt werden kann. Lernenden würde sich damit die Möglich- keit erschließen, systematisch auch nicht formale und informelle Lernformen zu praktizieren. Nicht formales und informelles Lernen sind anerkannte Bausteine, um Wissen nachhaltig zu festigen. Als Anwendungsbeispiel für die Lehrenden soll die Learning Community des EUProjektes »Community of Integrated Blended Learning in Europe (COMBLE) dienen, deren Gegenstand u. a. der Aufbau der wiki-basierten "Methopedia« ist, um 
Methoden des Lernens und Lehrens praxisorientiert mit Erfahrungswissen austauschen und darüber selbst lernen zu können.

\section{Virtuelle Communities nehmen Einzug in unser Leben}

Inwieweit virtuelle Communities Einzug in unser Leben halten, soll die folgende kurze Erzählung verdeutlichen: Als wir eine Freundin im Café trafen, stellte sie uns eine Bekannte vor, die einen Tag zuvor aus London angereist war. Nach 10 Minuten Unterhaltung fragte einer von uns, wo sie sich kennen gelernt haben. Ihre Antwort war: myspace. Es stellte sich heraus, dass beide Frauen Mitglieder einer Gruppe dieses sozialen Netzwerkes sind und sich für lernpsychologische Themen interessieren, die sie im Laufe von einigen Monaten online austauschten. Inzwischen lernen sie sich auch persönlich kennen. MySpace [1] ist eine von vielen »Communities«, in denen Millionen von Menschen sich in Profilen darstellen und sich untereinander elektronisch via Internet austauschen, unabhängig vom ursprünglichen Schwerpunkt, der bei MySpace im Bereich Musik liegt. In den letzten drei Jahren kam es zwar immer wieder zu öffentlicher Kritik wegen Sicherheitslücken, Profilfälschungen, Datenverkäufen u. a. m., doch das stört offenbar nicht wirklich. Weitere Beispiele solcher virtueller Communities bzw. sozialer Netzwerke sind Facebook, Flickr, Xing oder StudiVZ, die inzwischen für Millionen von Menschen ein fester Bestandteil ihres Lebens sind.

"Generation facebook" betitelte der stern seine Ausgabe am 03.09.2009, in der er diese sozialen Netzwerke und etliche Mitglieder, darunter auch bekannte Persönlichkeiten wie Renate Künast, Ursula EngelenKefer und Karl-Theodor zu Guttenberg, vorstellte. [stern 2009] »Facebook sammelt bis zu 90 persönliche Angaben, die jedes Mitglied freiwillig einstellen kann; zum Vergleich: Der Erfassungsbogen der Stasi zählte 48 persönliche Daten der Überwachten. « [ebenda: 132] Ist es nicht erstaunlich, wie freizügig die persönlichen Angaben getätigt werden? Ist es nicht umso erstaunlicher, als dass bekannt sein dürfte, dass solche »Datenspuren von und über Menschen im Internet nie vollständig gelöscht werden können? »Laut einer Dimap-Studie im Auftrag der Bundesregierung informiert sich mittlerweile jedes vierte Unternehmen in Netzwerken über Mitarbeiter und Bewerber.« [ebenda: 130] Und über Xing, das sich auf Geschäftskontakte spezialisiert hat, wird berichtet, dass in der Finanzbranche fast jeder, selbst die Vorstände, Mitglied seien. [ebenda:130] Über die Netzwerke des Internets »Freunde« zu finden, ist offenbar ein Alltagsphänomen geworden. Darüber hinaus geht es allerdings auch um's liebe Geld, wie es dem Interview mit dem Facebook-Gründer Mark Zuckerberg zu entnehmen ist: »Es wird in Zukunft darum gehen, dass die Firmen in den sozialen Netzwerken die Nutzer dazu bekommen, dass die ihren Freunden die jeweiligen Produkte empfehlen. Nichts bietet mehr Kaufanreiz als die persönliche Empfehlung eines Freundes.« [ebenda: 133] Mit Bezug auf den Bildungsbereich stellt sich dabei die Frage, wie das Phänomen der großen Akzeptanz sozialer Netzwerke im Sinne von Bildung und lebensbegleitendem Lernen genutzt werden kann und wie sich persönliches Engagement und Weiterempfehlungen sinnvoll in der Fort-, Aus- und Weiterbildung einsetzen lassen.

Doch was sind »Communities « und was macht sie für die Menschen so attraktiv? Der Begriff »Community« wird in der Regel synonym für den deutschen Begriff »Gemeinschaft« verwendet und bezeichnet keinesfalls ein neuartiges Phänomen des sozialen Zusammenschlusses. [Scholl et al. 2009] Bereits im 19. und frühen 20. Jahrhundert haben sich die Soziologen Ferdinand Tönnies und Max Weber mit dem Gemeinschaftsbegriff befasst und diesen über das Kriterium formaler Rationalität von »Gesellschaft« bzw. »Vergesellschaftung « als Gegenbegriffe abgegrenzt. Gemeinschaft bezeichnet in dieser Gegenüberstellung eine natürlich gewachsene, eher organische und von Traditionen und Gefühlen bestimmte Form des sozialen Zusammenschlusses, im Unterschied zur Gesellschaft als ihr rationales, kalkulierendes Gegenmodell [siehe Tönnies 1963 und Weber 1972]. An diesen frühen Gemeinschaftsbegriff knüpfen auch die jüngeren Definitionen »virtueller Gemeinschaften« an, die insbesondere den sozialen Zusammenschluss auf der Grundlage persönlicher Beziehungen im Cyberspace betonen. Die wohl bekannteste Definition stammt von Howard Rheingold: »Virtual communities are social aggregations that emerge from the Net when enough people carry on those public discussions long enough, with sufficient human feeling, to form webs of personal relationships in cyberspace.« [Rheingold 1993]

Tönnies ist noch vom Verfall aller Gemeinschaft ausgegangen, vom verdrängenden Vormarsch der Gesellschaft, die ihre rational-formalen Prinzipien an die Stelle gemeinschaftlicher Verbundenheit setzt. 
[Tönnies 1963] Im Begriff der virtuellen Gemeinschaft (virtuellen Community) jedoch scheint sich kurioserweise das Gegenteil abzuzeichnen, denn ausgerechnet der technologische Fortschritt, selbst ein Produkt rationaler Vergesellschaftung, gibt der vermeintlich verdrängten Gemeinschaft neue Impulse. Mit dem Internet und seinen neuen Kommunikationsformen wird der Gedanke der Gemeinschaft (die Community) nicht nur ins Netz, sondern auch in die reale Welt zurückgebracht. Der Begriff und auch seine Ausprägung ist somit nicht das eigentlich Neue, sondern eher das aus soziologischer Sicht interessante Phänomen, dass die Gemeinschaft und ihre Prinzipien auf der technischen Basis der weltweiten Vernetzung von Computern eine Renaissance erfährt.

Das Internet (der Gesellschaft) ist inzwischen zu einem Massenmedium (der Gemeinschaft) geworden und hat dabei vor allem die Kommunikation verändert. Dass sich mit seiner wachsenden Verbreitung die Nutzungsweise des Netzes selbst verändert hat, wird im Begriff des Web 2.0 deutlich. Das Augenmerk wird hier auf die interaktive Einbeziehung der Nutzer gelegt, die ihre bisher eher passive Betrachterrolle im Internet mit eigenen Beiträgen überwinden sollen. Diese aktivere Rolle des Nutzers findet seinen Ausdruck in dem von Alvin Toffler geprägten Begriff des Prosumenten, der Personen bezeichnet, die sich sowohl als Konsumenten (engl. consumer) als auch als Produzenten (engl. producer) der von ihnen genutzten Güter kennzeichnen lassen. [Toffler 1993] Dieser Wandel des Nutzers vom Konsumenten zum Prosumenten bedient sich einer Vielzahl von interaktiven Techniken und Anwendungen wie Internettagebücher (Blogs), kooperativ geschriebene Texte (Wikis), Empfehlungssysteme (durch Nutzerbewertung), neue Dienste zur kooperativen Erschließung von Wissen durch kooperative semantische Techniken (»folksonomies«), gemeinsame Diskussionen (Foren) sowie das gemeinsame Bereitstellen und Bewerten von Inhalten (z. B. Videos in YouTube, Fotos, Podcasts, Texte, Musik usw.). Mit der Nutzung solcher Dienste werden sich auch neue kulturelle Praktiken herausbilden sowie Lehr- und Lernprozesse beeinflusst. Neue Gruppenzusammenhänge, neue Vernetzungsformen, neue Arten der Unterstützung und der Zusammenarbeit formieren sich, in denen die Nutzer ihre eigenen Inhalte generieren, einstellen und austauschen. Diese interaktiven Strukturen und vielfältigen Kommunikationsformen via Internet werden von allen Nutzern und damit auch von Lernenden zusätzliche
Kompetenzen erfordern - ebenfalls ein Hinweis an den Bildungsbereich, solche Fertigkeiten und Fähigkeiten kritisch begleitend zu vermitteln. Das setzt allerdings voraus, dass die »Vermittler « solcher Kompetenzen, allgemein »Lehrende« genannt, selbst dazu in der Lage sind.

\section{Informelles Lernen}

ImUnterschiedzurtraditionellen Gemeinschaftkönnen sich virtuelle Gemeinschaften schneller und unabhängig von Ort und Zeit bilden. Über diese gemeinschaftsbildende Qualität moderner Kommunikationsmedien werden die virtuellen Gemeinschaften zu sozialen Netzwerken mit sozialen Interaktionen. Daher hat das Web 2.0 gerade keinen formalen, institutionellen und instruktionalen Charakter. Was sich im Ökonomischen als Wandel vom Konsumenten zum Prosumenten [Toffler 1993] ausdrückt, findet sich somit auch im Kontext des Lernens: Die Mitglieder der sozialen Netzwerke lernen aus der Praktik heraus, wie es funktioniert, miteinander Informationen zu organisieren, zu ergänzen, neu und in anderen Zusammenhängen zu nutzen, Inhalte co-produziert zu erstellen, miteinander elektronisch zu kommunizieren und miteinander zu partizipieren und zu lernen, damit gleichsam im Prozess das Lernen zu lernen. Gleichzeitig etabliert sich ein kollektives Eigentumsrecht an den gemeinsamen Ergebnissen. Darüber hinaus verschwimmt in den Communities die klare Trennung der Rollen als »Lernende « und »Lehrende « die Mitglieder sind beides in einer Person und zur gleichen Zeit.

Im Wiedererstarken des Community-Gedankens zeigt sich somit eine Entformalisierungstendenz für die Lösung von Problemen und für das Lernen. Lernen heißt nach Herbert Altrichter unter Bezug auf Jean Lave und Etienne Wenger ein »Sich-Einlassen auf die Welt« [Altrichter 2002]. »Lernen ist Tun, ist Handeln, ist eine Art, in der sozialen Welt zu sein, nicht bloß eine Art, Wissen über sie aufzubauen...«[ebenda: 202] »Lernen ist situiert: Lernend stehen AkteurIn und Welt in einer wechselseitigen, letztlich unaufhebbaren Abhängigkeit: Ohne konkreten Ort kann man sich Lernen nicht vorstellen. Lernen braucht ein Einlassen auf bestimmte soziale Situationen, es schöpft aus ihnen und es ist in einem gewissen Sinne auch an sie gebunden. " [ebenda] Lernen ist damit nicht das passive Aufnehmen von Lerninhalten, sondern »Tun«. Der moderne Ort - wo 
dieses Tun geschieht - wird zunehmend elektronisch über Lernplattformen oder virtuelle Umgebungen wie in Second Life abgebildet. Das Konzept des situierten Lernens ist gleichzeitig Antwort auf den Umstand, dass sich Wissen und Probleme immer weniger kontextunabhängig betrachten lassen. Selbstorganisation tritt an die Stelle von Instruktion und Anweisung. Die Lernenden werden aus ihrer Isolation herausgeholt und Lernen wird zunehmend zur Gruppenaktivität.

Dieser Veränderungsprozess ist auch daran erkennbar, dass das elektronische Lernen (E-Learning) im Laufe seiner Entwicklung zunehmend kommunikativer und interaktiver wird, sodass die Lernenden mehr und mehr durch die Begleitung und Betreuung von »E-Tutoren« bzw. »E-Mentoren« aus der Einsamkeit des Online-Lernens herausgeholt werden. Die Veränderungen zeigen sich daher ebenso in der Erweiterung des E-Learnings um handlungsorientierte und kontextbezogene Lernkonzepte wie »Blended Learning « [2], »Problembased « bzw. »Project-based Learning « [3] oder »Action Learning « [4] sowie in der wachsenden Vielfalt und Kommunikationsorientierung seiner technologischen Basis.

Diese Lernmodelle des Online-Lernens rücken immer stärker die Lernenden und ihre soziale Interaktion in den Mittelpunkt. Thomas Ryberg, dänischer Partner des von der Technischen Hochschule Wildau [FH] geleiteten EU-Projektes COMBLE [5], sieht die Zukunft des E-Learnings entsprechend im praxisorientierten Netzwerklernen personalisierter Lernumgebungen. [Ryberg 2009] Diese »Personal Learning Environments« (PLE) könnten unserer Meinung nach insbesondere für die praxisorientierten Fachhochschulen von Interesse sein, weil sich über das Netzwerklernen nicht nur neue Zielgruppen erschließen, sondern auch Wege für die Integration kulturell unterschiedlicher Praktiken aller Beteiligten aufzeigen lassen. Darüber hinaus ist im Zuge des demographischen Wandels noch ein großes Potenzial zu erschließen, denn es ist nach Untersuchungen davon auszugehen, dass europaweit ca. $43 \%$ aller Bürger bislang nicht an der »Wissensgesellschaft « partizipieren. [vgl. Jokisalo et. al. 2009]

Laut EU-Kommission hat sich »in den letzten Jahren (...) die Einsicht immer mehr durchgesetzt, dass Lernprozesse in nicht formalen und informellen $\mathrm{Zu}$ sammenhängen von maßgeblicher Bedeutung für die Umsetzung des Ziels >lebenslanges Lernen`sind." [Europäische Kommission 2001: 9] Daraus ergibt sich die Notwendigkeit neuer Strategien zur Ermittlung und
Validierung derartiger »unsichtbarer« Lernergebnisse. Auf europäischer Ebene werden die folgenden Definitionen für die unterschiedlichen Lernformen zugrunde gelegt:

- " Formales Lernen findet üblicherweise an Einrichtungen der allgemeinen oder beruflichen Bildung statt und weist strukturierte Lernziele, Lernzeiten und Lernförderung auf. Aus Sicht des Lernenden ist es zielgerichtet und führt zur Zertifizierung.

- Nicht formales Lernen findet nicht an einer Einrichtung der allgemeinen oder beruflichen Bildung statt und führt üblicherweise nicht zur Zertifizierung. Es ist jedoch intentional aus Sicht der Lernenden und weist strukturierte Lernziele, Lernzeiten und Lernförderung auf.

- Informelles Lernen findet im Alltag, am Arbeitsplatz, im Familienkreis oder in der Freizeit statt. Es ist nicht strukturiert und führt normalerweise nicht zur Zertifizierung. Es ist in den meisten Fällen nichtintentional aus Sicht der Lernenden.« [ebenda: 32f.]

Das Lernen, das in virtuellen Communities weltweit praktiziert und durch das Web 2.0 technologisch ermöglicht wird, ist in der Regel nicht formal bzw. informell und damit auch nicht instutionell organisiert oder strukturiert. Verschiedene Untersuchungen gehen davon aus, dass 70 - 90\% aller Lernaktivitäten informell verlaufen [vgl. Jokisalo et al. 2009]. Es ist daher zu erwarten, dass die Anzahl der sozialen Netzwerke und deren Funktionen zukünftig weiter steigen wird. Soziale Netzwerke und ihre Funktionen zeigen große Potenziale für das lebenslange Lernen. Netzwerklernen mit einem hohen Anteil an informellem Lernen könnte dabei eine wichtige Rolle gerade im beruflichen und privaten Bereich einnehmen.

Der Umgang mit den Social Communities wird damit auch im E-Learning zur wichtigen (Medien-)Kompetenz - so wie es der verantwortungsvolle Umgang mit den Fernsehprogrammen oder Computerspielen auch ist. Eigenverantwortung, Bedachtsamkeit im Umgang mit persönlichen Daten, die angemessene Umsetzung persönlicher und beruflicher Ziele sowie ein angemessenes Zeitmanagement werden dabei eine zentrale Rolle spielen. Die Hochschulen sollten diesen Trend bei der Entwicklung ihrer Lernarrangements ernst nehmen und für die Lehrenden und Lernenden Rahmenbedingungen schaffen, welche die Integration von nicht formalem und informellem Lernen in die formalen $\mathrm{Ab}$ läufe des formalen Lernens sicherstellt. 


\section{Was zeichnet lernende Communities aus?}

Das wesentliche Unterscheidungsmerkmal, das »Learning Communities« im Gegensatz zum traditionellen Lernen ausmacht, liegt darin, dass Lernen hier einen nicht formalen bzw. informellen Charakter aufweist. Und die Frage ist damit zunächst, welche Möglichkeiten Lehrende in ihren Institutionen haben, Social Communities für das Lernen zu nutzen. Grundsätzlich besteht für sie natürlich die Möglichkeit, Mitglied der Netzwerke zu werden, um dann eine solche Learning Community auszubauen. Unter dem Begriff der "Learning Community « wird seit einigen Jahren weltweit nach neuen Wegen in der Ausgestaltung der schulischen, universitären und beruflichen Aus-, Fort- und Weiterbildung gesucht. Ihre theoretischen Wurzeln haben die Learning Communities im Konzept des situierten Lernens. Im Verständnis dieses Ansatzes kann Wissen nur in einem aktiven und individuellen Konstruktionsprozess gewonnen werden. Wissen ist somit nicht kontextunabhängig und kann deshalb auch nicht einfach von einer Person zu einer anderen Person übertragen werden. Die Kontextbezogenheit des Wissens mündet in der Forderung, dass die Lernsituation den späteren Anwendungssituationen entsprechen und dass Lernen und Arbeiten in Gruppen unter Berücksichtigung der Anwendungsbedingungen erfolgen muss. Hier finden sich bereits die Anlagen einer Learning Community, die in der Definition von Seufert eine Lern(er)gemeinschaft darstellt, »[...] in der Personen zusammengeschlossen sind, die sich gemeinsam mit einem bestimmten Thema intensiv auseinandersetzen wollen, gemeinsam lernen, schon vorhandenes Wissen austauschen und gemeinsam an Problemstellungen arbeiten« [Seufert 2004: 30].

In Abgrenzung zu anderen Formen von Communities, wie z. B. den von Lave und Wenger [1991] beschriebenen »Communities of Practice« $(\mathrm{CoP})$, lassen sich Learning Communities durchaus in den Bereich des formalen Lernens im Rahmen einer Bildungsmaßnahme einordnen. Indiz dafür ist, dass eine solche Community sich nicht frei zusammen findet, sondern durch die Rahmenbedingungen der Institution (z. B. Schule, Universität) im Anfangsstadium stark instruktional (z. B. durch Lehrer, Professoren) geführt und geprägt wird [vgl. Seufert 2004: 30]. Die Lehrpersonen übernehmen innerhalb der Learning Community, zumindest in der Anfangsphase, eine führende Rolle, die den Lernenden hilft, sich in der neuen Lernwelt zurecht zu finden. Die Learning Community der Institution findet sich damit nicht selbstgesteuert zusammen, sondern wird institutionell, formal eingerichtet. Seufert [2004] sieht dennoch die Möglichkeit, die Lernenden untereinander zu vernetzen und das informelle Lernen zu unterstützen. Dalsgaard [2006] unterscheidet folgende drei Möglichkeiten des netzbasierten Lernens:

- Zusammen arbeiten (z. B. für einen Vortrag oder eine Seminararbeit),

- den gleichen Kontext teilen (z. B. Seminar- oder Arbeitskollegen tauschen sich aus),

- ein gleiches Interesse haben (z. B. eine Sprache lernen).

Auch an dieser Stelle zeigt sich die Notwendigkeit, die Lehrenden selbst mit Kompetenzen auszustatten und institutionell zu unterstützen, damit sie an der Learning Community teilhaben können.

\section{Anwendungsbeispiel Methopedia aus dem EU-Projekt COMBLE}

Lebt die Qualität eines Seminars vom Engagement der Lehrenden? Von ihren didaktischen Konzepten oder von der organisatorischen Unterstützung, die sie erhalten? Wieviel Idealismus können sich Lehrende erhalten? Welche andere Formen der Motivation für internetgestütztes Lernen können aktiviert und wie genutzt werden? Mit solchen Fragen beschäftigt sich das von der EU geförderte und von der TH Wildau [FH] geleitete COMBLE-Projekt, dessen Ziele und Produkte in den Wissenschaftlichen Beiträgen 2008 dargestellt wurden. [Niemczik et al. 2008] Sein oberstes Ziel ist,

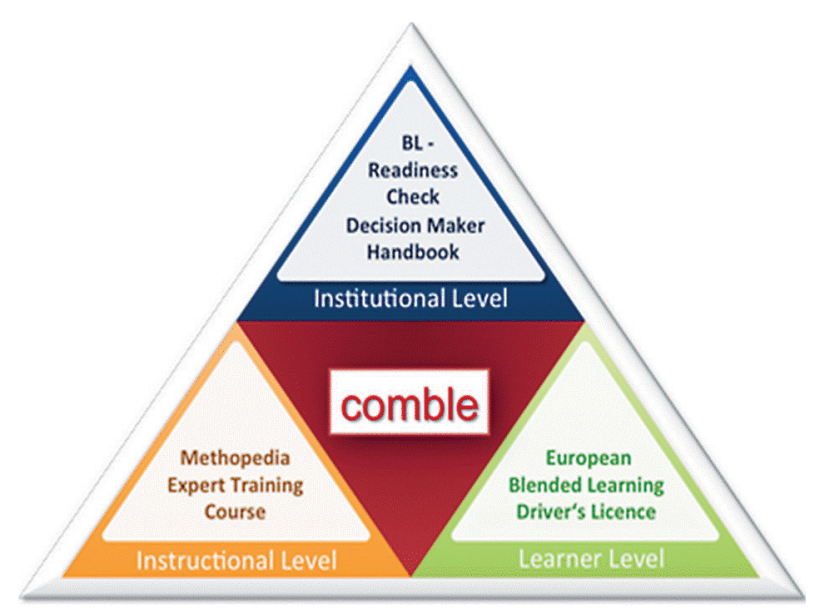

Abb. 1: Symbolische Darstellung der im COMBLE-Projekt untersuchten drei Ebenen (institutional, instructional and learner level) und ihre Produkte 
Blended Learning ganzheitlich $\mathrm{zu}$ fördern. COMBLE befindet sich inzwischen in der zweiten Hälfte der Projektverwirklichung und wurde in der Zwischenprüfung sehr positiv bewertet. Auf jeder der drei untersuchten Ebenen sind spezifische Produkte entstanden bzw. noch in der Entwicklung, wie es Abb. 1 symbolisch darstellt. Innerhalb dieses Beitrags interessiert insbesondere das »Instructional Level« aus Abb. 1 und dessen Produkte Methopedia und Expert Training Course.

Was ist Methopedia? Methopedia [6] ermöglicht den wiki-basierten Austausch zu Methoden und Lernansätzen (Pedagogical Approaches). Mit der Wiki-Software wird auf eine Technologie des Web 2.0 zurückgegriffen, die auf Kooperation und Austausch angelegt ist. Jeder Lehrende, der diese Technologien verwenden will, muss sich auf didaktische Ansätze und offene Seminarmethoden einlassen, die dem konstruktivistischen Lernparadigma folgen (ohne dies zwingend zu wissen). In konstruktivistischer Lesart ist Lernen gerade nicht das passive Aufnehmen von Lerninhalten, sondern das
Tun, das bereits behandelt wurde. Die Lehrenden wissen jedoch oft nicht, was diese Ansätze bedeuten und wie sie erfolgversprechend umzusetzen sind. Mit Methopedia erhalten Lehrende neue Inspirationen und können Methoden wie Expertenlernen, Wikiarbeit, Webquests, Pro- und Kontraanalyse, Gruppenarbeit, Tandem-Lernen, Open Space, Video casts, Gruppenpuzzle etc. in Form eines Wikis für die Gestaltung der eigenen Seminare verwenden oder anpassen (vgl. Abb. 2 mit der Methode »Brainwriting «). Gleichzeitig können die Lehrenden ihre Erfahrungen mitteilen und in der Social Community Methodpedia diskutieren. Darüber hinaus wird ein unter »Creative Commons « verfügbarer Expert Training Course, der derzeit u. a. in Second Life mit Lehrenden aus Deutschland, Dänemark, Polen, Estland und Argentinien getestet und mit einem Zertifikat abschließen wird, die Lehrenden im Kennenlernen von konstruktivistischer Didaktik in Form von problembasiertem Lernen unterstützen.

\section{Meth pedia - Brainwriting}

\begin{tabular}{|l|}
\hline Explore \\
\hline Homepage \\
\hline Search \\
\hline Sorted titles \\
\hline Most visited \\
\hline Brainwriting \\
\hline Contribute \\
\hline Login \\
\hline
\end{tabular}

\section{Short description}

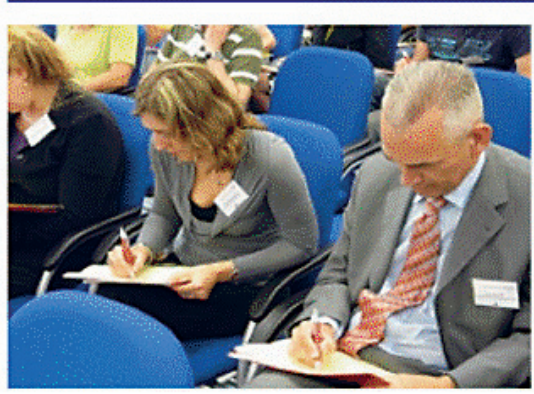

Brainwriting is also called the "6-3-5 method": 6 people, 3 ideas each, and 5 rounds. Basically, the same rules apply as in brainstorming. The difference is that in brainwriting the creative phase consists of writing instead of talking. Everybody first writes down their own ideas and then amends the ideas of all other participants in turn. Finally, possible solutions are discussed with regard to their fit to the problem at hand.

\section{Process description}

1. The problem is defined.

2. 6 participants generate 3 new ideas each.

3. Notepads are passed around in a circle to the next person.

4. Each person complements or amends the ideas of his neighbor.

5. The cycle is continued $\mathbf{5}$ times.

6 . Conclusions and solutions are being created. 


\section{Was sind Methoden und Lernansätze?}

Innerhalb der Learning Community des COMBLE-Projektes entstanden aufgrund der unterschiedlichen und auch kulturell geprägten Sichtweisen Diskussionen zu Methoden. Beschreiben Methoden eine Lernaktivität eines Einzelnen oder einer Gruppe (Stationenlernen, Case study etc.) innerhalb eines begrenzten Zeitraums (eine Stunde bis zwei Tage) oder versteht man darunter einen generellen Lernansatz, wie es z. B. problembasiertes, sozial-konstruktives oder netzwerk-unterstütztes Lernen ist? Diese Fragestellungen zeigen, dass in Learning Communities zwischen allen Beteiligten auch Begriffsklarheit hergestellt werden muss. Hinsichtlich eines möglichen Seminardesigns wird im Projekt ein Ebenen-Modell genutzt, das von Berge [2006: 96] adaptiert wurde (siehe Abb. 3):

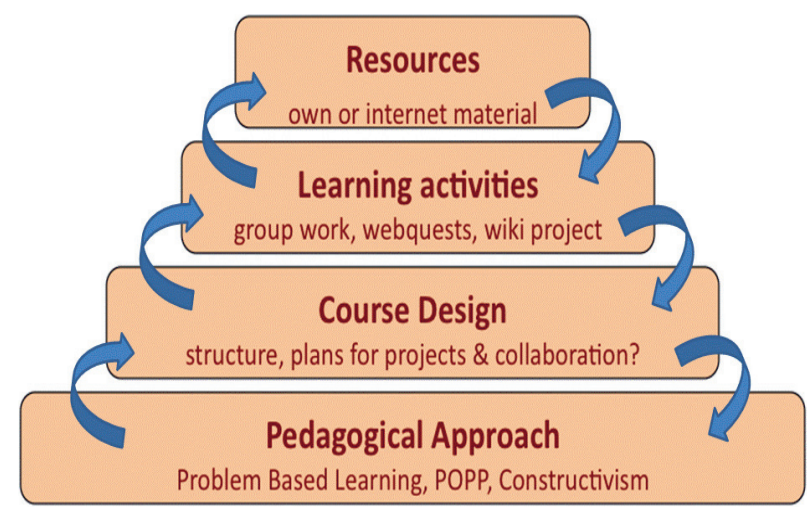

Abb. 3: Pedagogical design levels

Ein genereller Lernansatz (»Pedagogical Approach «) könnte z. B. konstruktivistischer Natur sein: Die Lernenden suchen sich selbstständig und in Gruppen Probleme, die sie innerhalb eines Projektthemas lösen wollen. Das selbst gesuchte Problem fordert die Studierenden dabei so heraus, dass sie gewillt sind, Zeit und Engagement zu investieren. Das Lernen an sich findet demnach in Form eines Problemlösungsprozesses statt. Dieser Ansatz eines Problemlösungsprozesses entspricht der »Problem-Oriented Project-Pedagogy" (POPP). POPP ist eine moderne, konstruktivistische Lernmethode, die von den dänischen Partnern des COMBLE-Projektes entwickelt wurde und an der Universität Aalborg in allen (!) Studiengängen eingesetzt wird. Die Merkmale von POPP werden als »Aalborg Model of Problem-Based Blended Learning « wie folgt beschrieben [Aalborg University 2009]:
- »Problem-Oriented Project-Pedagogy as organizing principle.

- Problem orientation indicates an open frame, where participants formulate problems (as opposed to predefined problems set by the instructor or textbook).

- Project pedagogy indicates that participants are expected to work on long-term openended projects (as opposed to short-term, narrow assignments).

- Learning is realized in a community of learners organized in small interdependent groups «.

Die praktischen Erfahrungen solcher Lernprojekte lassen den Schluss zu, dass generelle Lernansätze von der jeweiligen Institution durch geeignete Rahmenbedingungen unterstützt werden müssen. Denn ein wie hier beschriebenes Seminar durchbricht das 90-MinutenSchema von Unterrichtseinheiten, es fordert eine andere Art der Bewertung der Ergebnisse und die Lehrenden/Dozenten erfahren einen Rollenwechsel, weg vom Vermittler hin zum Unterstützer, mit anderen Vorbereitungs-, Durchführungs- und Nachbereitungszeiten, ggf. werden auch andere Räumlichkeiten benötigt.

Die Art des Lernansatzes hat Einfluss auf das Kursdesign, das in Abb. 3 als nächste Ebene dargestellt ist. Wie soll das Lernen absolviert werden, eher lehrer- oder eher lernerzentriert? Gibt es im Kursdesign Projekte, an denen die Lernenden/Studierenden arbeiten oder wird der Kurs eher vorlesungsartig abgehalten?

Die Komponenten des Kursdesigns sind die Lernaktivitäten, in Abb. 3 als dritte Ebene vermerkt, die durch die Methoden unterstützt werden können. Die Methoden haben unterschiedliche Ziele, wie z. B. soziales Lernen, Reflexionen, Zusammenarbeit oder Tiefenverstehen, zu unterstützen. Eine interessante Perspektive auf die Umsetzung solcher Methoden sind so genannte »collaboration scripts «: »... a set of instructions relating to how group members should interact, how they should collaborate and how they should solve a problem. When a teacher engages students in collaborative learning, he or she usually provides them with global instructions such as >do this task in groups of three « " [O’Donnell und Dansereau 1992] Ein solches Skript ist eine Art Vertrag zwischen den Lehrenden und Lernenden, der die Art der Zusammenarbeit festlegt. [Dillenbourg 2002] Bezug nehmend auf die letzte oberste Ebene des in der Abb. 3 dargestellten Modells müssen auch die Lernmaterialien den tieferen Ebenen entsprechen, da sie Teil der Methoden sind. 


\section{Wie kann man mit Methopedia arbeiten?}

Zunächst finden sich viele schon beschriebene Methoden in Deutsch oder Englisch im Wiki wieder (Abb. 4), die sich für eigene Seminare nutzen lassen. Die Methoden lassen sich verändern und kommentieren. Außerdem können die Autoren eines Artikels über deren Zugehörigkeit zu weiteren Netzwerken wie Facebook oder LinkedIn kontaktiert werden.

Für Lehrende bzw. Dozenten in Fort-, Aus- und Weiterbildung, die ihre Erfahrungen einbringen und austauschen wollen, finden sich verschiedene Vorlagen, in denen eine neue Methode oder ein Lernansatz beschrieben und bearbeitet werden können (Abb. 5).

Jede Methode kann mit Metadaten angereichert werden, um später eine sinnvolle Suche zu gewährleisten. Dies wird in Abb. 6 verdeutlicht. Die Hauptgruppen der Metadaten beziehen sich auf die Anzahl der Lernenden, die Zielgruppen, die Lernphase, die Lernform und den Lerngegenstand.

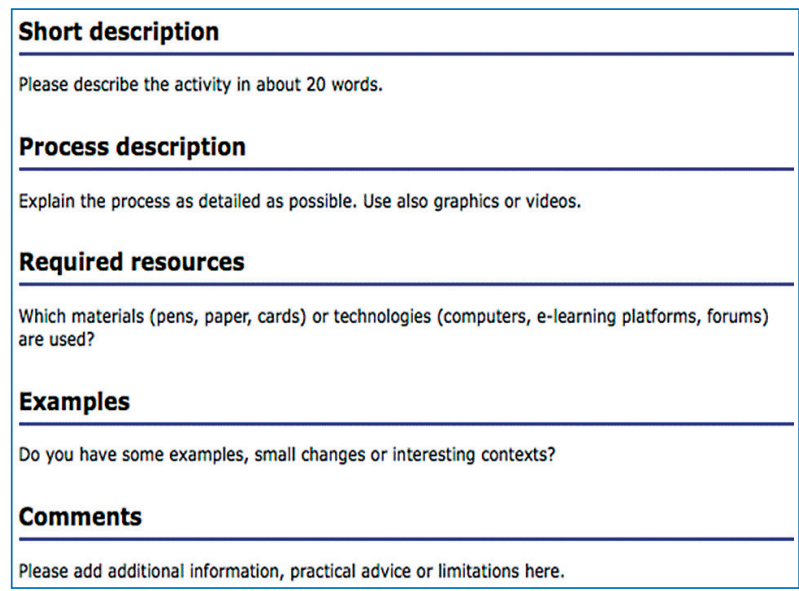

Abb. 5: Vorlage zur Beschreibung einer Methode in Methopedia

\begin{tabular}{|c|c|c|c|c|}
\hline $\begin{array}{l}\text { Number of learners } \\
\square \text { Single }\end{array}$ & $\begin{array}{l}\text { Target Groups } \\
\nabla \text { University }\end{array}$ & $\begin{array}{l}\text { Timeline } \\
\square \text { Beginning }\end{array}$ & $\begin{array}{l}\text { Settings } \\
\Downarrow \text { Face to face }\end{array}$ & $\begin{array}{l}\text { Subjects } \\
\square \text { Biology }\end{array}$ \\
\hline$\square$ Partner & $\square$ Business & (V) Main learning phase & $\square$ Online & Psychology \\
\hline$\nabla$ Group & Further education & $\square$ End & $\square$ synchronous & Dathematics \\
\hline$\square$ Large group & 『vocational education & & $\square$ Asynchronous & $\nabla$ other \\
\hline
\end{tabular}

Abb. 6: Metadaten zur Methodenbeschreibung in Methopedia

\section{Methofia - TitleIndex}

\begin{tabular}{|l|}
\hline Explore \\
\hline Homepage \\
\hline Search \\
\hline Sorted titles \\
\hline Most visited \\
\begin{tabular}{|l|}
\hline Page \\
\hline Immutable Page \\
\hline Discussion \\
\hline Revision History \\
\hline Subscribe \\
\hline Attachments \\
\hline More Actions: \\
\hline \\
\hline Contribute \\
\hline Add Page \\
\hline Settings \\
\hline Logout \\
\hline
\end{tabular}
\end{tabular}

This is an index of all pages in this Wiki.

See also:

- WordIndex -- a permuted index of all words occuring in titles

- SiteNavigation -- other indexing schemes

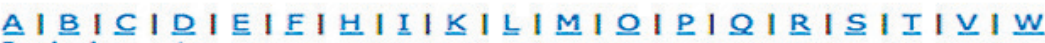
Include system pages

A

- $A B C L i s t e[0]$

- AddPage

- Assoziationskettell

- Aufgaben delegieren(1)

B

- BilderMalend

- Blitzlicht!l

- Brainstorming(1)

- Brainwriting@

- Brief an mich selbstt! 
Innerhalb des EU-Projektes COMBLE sind verschiedene Videoanleitungen entstanden, so auch eine genaue Videoanleitung für das Erstellen von Methoden und Lernansätzen innerhalb von Methopedia, die der Webseite von COMBLE zu finden ist. [7] Weiterhin arbeitet das Projektteam an einem Seminarplaner, der das Integrieren von neuen Methoden in eigene Seminare für die Dozenten vereinfachen soll. Der »Methopedia-Learning Design-Planer« benutzt Methopedia als Methodenpool für das Gestalten eines Seminardesigns und ist in der Abb. 7 dargestellt.

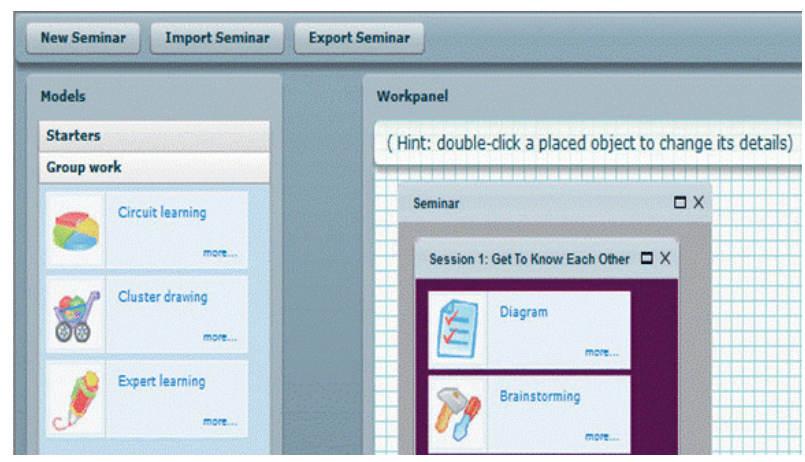

Abb. 7: Methopedia-Learning Design-Planer zur Entwicklung des eigenen Kursdesigns

\section{Fazit und Ausblick}

Lernen in Communities unter Nutzung von Web 2.0 Technologien ist eher nicht formal und informell und bietet große Chancen für das Anstoßen von lebenslangen Lernprozessen in nicht formalen und informellen Zusammenhängen. Learning Communities könnten sich als sehr bedeutsam in der Umsetzung der Zielsetzung des lebenslangen Lernens auch in formalen Lernprozessen erweisen. Die große Akzeptanz, die die Communities weltweit erfahren, sollte im Bildungsbereich ernst genommen und verstärkt genutzt werden. Die Lerntechnologien und damit auch die Learning Communities haben sich in den letzten Jahren rasant entwickelt und bieten ein großes Potenzial, die Qualität des Lehrens und Lernens zu verbessern. Jedoch werden sie nur dann ihre Wirkung entfalten, wenn die Institutionen passende Lernkulturen und Methodologien einführen helfen und wenn die Lehrenden aktives Lernen für die Studierenden ermöglichen. Entspechend merkt die Europäische Kommission im Progess Report »The use of ICT to support innovation and lifelong learning for all « an: »New innovative pedagogical and didactical approaches are needed to take into account the future learning needs and changing skills and competences necessary for employment, self-development and participation in a knowledge-based, digital society.« [European Commission 2008: 12]

Als Konsequenz werden formale Lernarrangements zukünftig zunehmend online-basiertes, nicht formales bzw. informelles Lernen in Form von sozialen Netzwerken berücksichtigen müssen. Lernen braucht ein Einlassen auf bestimmte soziale Situationen. Wie die Bindung an eine virtuelle Community vor allem durch persönliche Beziehungen der Mitglieder untereinander, sollten Learning Communities auch im formalen Rahmen dem nicht formalen und informellen Lernen mehr Raum geben. Learning Communities können am Anfang eher formell durch Lehrende eingerichtet werden, um später dem initiierten informellen Charakter durch die Lernenden ausfüllen zu lassen. Darüber hinaus kann eine Institution unterstützende Top-down Rahmenbedingungen mit Zertifizierung für ihre Lehrenden einführen, so, dass diese praktische Kompetenzen für ein nicht formales bzw. informelles Lernen der Lernenden im Bottom-up Prozess aktiv initiieren wird.

Doch die Lernenden/Studierenden werden ebenfalls gefordert. Ein wichtiger Schritt, um die pädagogische Innovation zu unterstützen, sind aktive, lernerzentrierte Methoden: »The reviewed studies showed that learner-centred guidance, group work and inquiry projects result in better skills and competencies and that interactive forms of e-learning can lead to a more reflective, deeper and participative learning, learning-by-doing, inquiry learning, problem solving, creativity, etc all play a role as competencies for innovation...« [ebenda: 11] Die unterschiedlichen Lernkulturen innerhalb von Institutionen - besonders im europäischen Kontext - bevorzugen entsprechend eine weite Variation von Lernansätzen. Forschungsstudien wie Pisa zeigen, dass sowohl Lerninstitutionen als auch Lehrende am Wissen um Lehrqualität teilhaben wollen. [Geller et al. 2007] Gerade hierfür ist es sinnvoll, Kommunikationsstrukturen auf Peer-to-Peer-Ebene für einen Austausch anzubieten. In der vorgestellten Methopedia-Plattform des EU-Projektes COMBLE wird dies zurzeit umgesetzt.

\section{Anmerkungen}

[1] http://www.myspace.com/, Zugriff am 16.09.2009.

[2] Siehe zum Beispiel Heinze, A. und Ch. Procter: Reflections On These Of Blended Learning, Conference Proceedings, Education in a Changing Environment, 13.-14. September 2004, published by the University of Salford. 
[3] Siehe zum Beispiel De Graaff, E. und A. Kolmos (Hrsg.) (2007): Management of Change - Implementation of Problem-Based and Project-Based Learning in Engineering, Sense Publishers, Rotterdam/Taipei.

[4] Siehe zum Beispiel Special Issue (1998): Action Learning, Performance Improvement Quarterly, Volume 11, Number 1, 1998.

[5] Community of Integrated Blended Learning in Europe, http:// comble-project.eu.

[6] http://www.methopedia.eu.

[7] http://comble-project.eu/Plone/methopedia-1/methopedia.

\section{Literaturverzeichnis}

Aalborg University (2009): General Course Description »Blended Learning Expert Training Course «, COMBLE Project, Grant Agreement Number 2007-3623/001-001.

Altrichter, H., Jean Lave und Etienne Wenger (2002): Die soziale Situierung professionellen Handelns. In: G. Breidenstein, A. Combe, W Helsper und B. Stelmaszyk (Hrsg.), Forum Qualitative Schulforschung 2, Leske + Budrich, Opladen.

http://books.google.de/books?id=4MR8DokR7XwC\&pg=PA202\&lpg= PA202\&dq=Lave,+Wenger, $+1991 \&$ source=bl\&ots=c_7aWP1alY\& sig=J_9d4I3FBUEwrW8bdg8Agz4peVM\&hl=de\&ei=oVWdSoqXM cGN_AbiiZTtAg\&sa=X\&oi=book_result\&ct=result\&resnum=9\#v= onepage\&q=Lave\%2C\%20Wenger\%2C\%201991\&f=false, Zugriff: 01.09.2009.

Berge, O. (2006): Reuse of Digital Learning Resources in Collaborative Learning Environments. PhD thesis, Faculty of Mathematics and Natural Sciences, University of Oslo.

Dalsgaard, Ch. (2006): Social software: E-learning beyond learning management systems, European Journal of Open, Distance and E-Learning (EURODL), ISSN 1027-5207, http://www.eurodl.org/ materials/contrib/2006/Christian_Dalsgaard.htm, Zugriff am 16.09.2009.

Dillenbourg, P. (2002): Over-scripting CSCL: The risks of blending collaborative learning with instructional design. In P. A. Kirschner (Ed). Three worlds of CSCL. Can we support CSCL (pp. 61-91). Heerlen, Open Universiteit Nederland.

Europäische Kommission (2001): Mitteilung der Kommission - Einen europäischen Raum des Lebenslangen Lernens schaffen, Generaldirektion Bildung und Kultur, Generaldirektion Beschäftigung und Soziales, 9, 32f.

European Commision (2008): The use of ICT to support innovation and lifelong learning for all - A report on progress. [Online] Retreived August 31, 2009 from http://ec.europa.eu/education/ lifelong-learning-programme/doc/sec2629.pdf.

Geller, C.; Olszewski, J.; Neumann, K. \& Fischer, H. E. (2007): Unterrichtsqualität in Finnland, Deutschland und der Schweiz: Merkmale der Tiefenstruktur von Physikunterricht und der Zusammenhang zur Leistung, in: D. Höttecke (Hrsg.), Kompetenzen, Kompetenzmodelle, Kompetenzentwicklung. Gesellschaft für Didaktik der Chemie und Physik. Jahrestagung in Essen 2007, LIT Verlag Berlin-Hamburg-Münster.

Jokisalo, E.; Riu, A. (2009): Informal learning in the era of Web 2.0. ICT and lifelong learning for a creative and innovative Europe Findings, reflections and proposals from the Learnovation project, http://www.elearningeuropa.info/files/media/media19656.pdf, Zugriff am 15.09.2009.

Lave, J.; Wenger, E. (1991): Partnerships as communities of practice / learning communities, http://www.learning-theories.com/ communities-of-practice-lave-and-wenger.html, Zugriff am 04.09.2009.

Niemczik, Ch.; Brenstein, E.; Scholl, M. (2008): COMBLE: European Community of Integrative Blended Learning Experts, Wissenschaftliche Beiträge 2008 der TFH Wildau, 87-93.

O'Donnell, A. M.; Dansereau, D. F. (1992): Scripted cooperation in student dyads: A method for analyzing and enhancing academic learning and performance. In: Hertz-Lazarowitz, R. and N. Miller (Eds.), Interaction in cooperative groups: The theoretical anatomy of group learning (pp. 120-141). London: Cambridge University Press.

Rheingold, H. (1993): The virtual community: homesteading on the electronic frontier. Reading Mass.: Addison-Wesley Pub. Co.

Ryberg, T. (2009): 3rd Generation PBL and Networked Learning. Skript zur Second Life Vorlesung am 14.09.2009, 20:00-22:00 Uhr, innerhalb des »Blended Learning Expert Training Course « des EU-Projektes COMBLE.

Scholl, M.; Niemczik, Ch.; Büschenfeldt, M. (2009): Die Rolle von Communities im E-Learning. Proceedings zur Tagung »Baltic Sea Forum 09 E-Learning « an der FH Stralsund, Stralsund, 05.10.2009, in Druck.

Seufert, S. (2004): Virtuelle Lerngemeinschaften: Konzepte und Potenziale für die Aus- und Weiterbildung, in: Zinke, G.; Fogolin, A. (Hrsg.): Online-Communities - Chancen für Informelles Lernen in der Arbeit, Bielefeld, 28-38.

Stern (2009): Osterkorn, Th. (Verantw.), Generation feacebook - Wie sich Millionen Deutsche im Internet vernetzen, Nr. 37 vom 3. September, Gruner und Jahr.

Toffler, A. (1993): Machtbeben: der globale Vorstoß der Informationseliten. Düsseldorf: ECON.

Tönnies, F. (1963): Gemeinschaft und Gesellschaft, Grundbegriffe der Reinen Soziologie. Darmstadt: Wissenschaftliche Buchgesellschaft.

Weber, M. (1972): Wirtschaft und Gesellschaft. 5. Aufl., Tübingen: Mohr Siebeck.

\section{Autoren}

\section{Prof. Dr. Margit Scholl}

TH Wildau [FH]

FB Wirtschaft, Verwaltung und Recht

Wirtschafts- und Verwaltungsinformatik

Tel. +493375 508-917

margit.scholl@tfh-wildau.de,www.tfh-wildau.de/scholl

Christian Niemczik, M. A. (Erziehungswissenschaftler) TH Wildau [FH] FB Wirtschaft, Verwaltung und Recht Projektmitarbeiter EU-Projekt COMBLE (Community of Integrated Blended Learning in Europe) Tel. +49 331 20029717, christian.niemczik@sudile.com http://www.sudile.com, http://comble-project.eu

\section{Dipl.-Soz. Maika Büschenfeldt}

TH Wildau [FH]

FB Wirtschaft, Verwaltung und Recht

Projektmitarbeiterin Projekt learn@wildau

(Zentrale Lernplattform Moodle)

Tel. +49 3375 508-379, maika.bueschenfeldt@tfh-wildau.de https://elearning.tfh-wildau.de/login/index.php 